\title{
Ciudades inteligentes, más que tecnología
}

Bibiana María Guerra de los Ríos*

Universidad del Rosario

bibiguerra@hotmail.com
Revista Cultura Económica

Año XXXVIII • N 100

Diciembre 2020: 39-65

DOI: https://doi.org/10.46553/cecon.38.100.2020.p39-65

Resumen: El concepto de ciudad evoluciona y se adapta a lo largo de la historia de acuerdo con el contexto dinámico mundial. Es así como actualmente las inversiones y los esfuerzos se están enfocando hacia el desarrollo de las ciudades inteligentes. Si bien en este tipo de ciudades la tecnología es un sector de suma importancia para el mejoramiento de la calidad de vida de los ciudadanos, esta es más un medio que un fin en sí mismo. Entre los componentes que se analizan dentro de este concepto multidimensional están la economía circular y el desarrollo sostenible, los datos, la idea de ciudades nocturnas, y la gobernanza y la participación ciudadana. Hoy en día, las decisiones más importantes se toman en los niveles locales pues es en el territorio desde donde los ciudadanos conocen sus necesidades más apremiantes y se localizan los problemas más latentes. Actualmente, las ciudades son quizás más relevantes que las mismas naciones a la hora de dar respuesta a los distintos problemas. Por lo anterior, las ciudades inteligentes surgen como posible solución a dichos fenómenos.

Palabras clave: Ciudades inteligentes; Ciudades nocturnas; Economía circular; Participación ciudadana; Tecnología

\section{Smart cities, more than just technology}

Abstract: The concept of city evolves and adapts throughout history according to the dynamic world context. This is how currently investments and efforts are being focused on the development of smart cities. Although in these types of city's technology is a very important sector for improving the quality of life of citizens, it is more a means than an end. Among the components that are analyzed within this multidimensional concept are the circular economy and sustainable development, data, the idea of nocturnal cities, and governance and citizen participation. Nowadays, the most important decisions are taken at the local levels, since it is in the territory from where citizens know their most pressing needs and the most latent problems are located. Currently, cities are perhaps more relevant than nations themselves when it comes to responding to different problems. Therefore, smart cities emerge as a possible solution to these phenomena.

Keywords: Smart cities; Nocturnal cities; Circular economy; Civic participation; Technology

* Recibido: 17/09/2020 - Aprobado: 16/11/2020 
"El siglo XIX fue el siglo de los imperios, el siglo XX fue el siglo de los estado-nación.

El siglo XXI será el siglo de las ciudades" Wellington E. Webb, Ex alcalde de Denver, Colorado, EE.UU.

\section{Introducción}

Si antes los países dominaban el mundo, con la globalización el poder ha descendido a niveles más bajos, con la ciudad a la cabeza. Aunque los problemas sean globales, las soluciones son locales, de ahí la relevancia que han adquirido estos territorios a nivel mundial. La Organización de Naciones Unidas (ONU) afirma que a 2050, 68\% (2.5 billones) de la población mundial será urbana, es decir, vivirá en las ciudades (Rodríguez, 2019: 4). De esta forma, las ciudades seguirán siendo relevantes porque deberán satisfacer una demanda cada vez mayor generada por una presión por los recursos inmensa. Asegura también la ONU que, a pesar de que las ciudades ocupan solo el $3 \%$ de la tierra, producen el $80 \%$ del producto interno bruto mundial. A su vez, "representan entre el 60\% y el $80 \%$ del consumo de energía y el 75\% de las emisiones de carbono" (Organización de Naciones Unidas, 2015: 1). Las ciudades han adquirido tanta preponderancia que el objetivo \#11 de los Objetivos de Desarrollo Sostenible (ODS) pactados en la Conferencia de Río+2O en 2012 se refiere a las "ciudades y comunidades sostenibles". El ODS 11 es lograr a 2030 que las ciudades y los asentamientos humanos sean inclusivos, seguros, resilientes y sostenibles, pues, aunque su desarrollo ha traído progreso a la gran mayoría de personas, todavía hay mucha pobreza e inequidad. Si bien el número de megaciudades ha crecido de forma abrupta en los últimos años, la idea hoy, y sobre todo con lo que sucede en el mundo a causa de la pandemia generada por el COVID-19, es que las ciudades intermedias deberían primar. Las ventajas de las grandes urbes son indudables; gran densidad poblacional, demanda de trabajo importante, oferta cultural innegable, etc. Sin embargo, un crecimiento acelerado y, en ocasiones sin planificar, ha hecho que las ciudades se conviertan en focos de polución, congestión y pobreza. De ahí que la mirada se gire a ciudades más pequeñas que, con una oferta similar de bienes y servicios, brinden mejor calidad de vida a sus ciudadanos.

La globalización, en la era moderna, ha dado lugar a transformaciones y avances en todo tipo de materias. Hoy se habla de ciudades sostenibles e inteligentes, aquellas donde se deben aprovechar los 
recursos naturales de forma eficiente y donde la tecnología juega un papel importante en su desarrollo. Aunque hoy en día no son tan innovadores como hace unos años, estos conceptos resultan prometedores para lograr la estabilidad y garantizar recursos a futuro. Si antes los gobiernos concentraban sus esfuerzos en lograr un mayor desarrollo económico, hoy el camino es el desarrollo sostenible, en el cual las tres dimensiones; económica, social y ambiental, interactúan para garantizar recursos a mediano y largo plazo de forma que las generaciones futuras cuenten también con recursos suficientes y de calidad. Por tanto, "son las ciudades los espacios para el desarrollo de los valores y los comportamientos democráticos, ya que el fenómeno de la globalización exige una respuesta ante los cambios de una sociedad más compleja, plural y diversa en el contexto de la gobernanza urbana" (Romero, 2018: 31).

Este artículo busca dar respuesta a las necesidades cambiantes de los ciudadanos mediante el concepto de ciudades inteligentes y varios de sus componentes más relevantes. El rápido crecimiento demográfico y los procesos de urbanización a nivel global han provocado el aumento en la demanda de los recursos y las necesidades de los habitantes. Como consecuencia de estos cambios rápidos, el mundo y su oferta no se han adaptado de la misma forma, conllevando a grandes desafíos y retos a nivel global. Los siguientes son algunos de los conceptos e ideas que pretenden dar solución a dichos retos, todos basados en el gran concepto del desarrollo sostenible.

Por un lado, la economía circular buscar aprovechar el concepto de sostenibilidad y cambiar la forma en que se producen las cosas, pasando de un modelo lineal a uno circular. Por su parte, la dinámica actual de las ciudades ha dado pie a que la actividad nocturna sea de gran valor para la economía mundial, especialmente en las grandes ciudades. De ahí, que incluso se hayan designado alcaldes nocturnos que velen para que la actividad de la noche sea un éxito. En esta misma vía, la participación ciudadana se ha convertido también en otro motor actual de las ciudades que ha permitido la realización de un mayor número de proyectos exitosos. En la actualidad los ciudadanos tienen mayor acceso a la información y se sienten empoderados para participar en política y en la construcción de ciudad. Finalmente, no se puede hablar de ciudades inteligentes sin tener en cuenta el factor tecnológico. Dichas herramientas son fundamentales para que las ciudades se adapten día a día a las necesidades ciudadanas y faciliten los estilos de vida. 
Este artículo se escribe en plena crisis mundial generada por la pandemia del Coronavirus (COVID 19), de ahí que ahora más que nunca las ciudades deben replantear su modelo de desarrollo y crecimiento, pues si bien son el foco principal del virus también son el lugar de sus soluciones. Los cambios que se desprenden de esta pandemia se basarán en soluciones donde los modos de vida sostenibles, el ser humano entendido a cabalidad como ciudadano, la micro movilidad y la atención al cambio climático deben primar.

\section{Las ciudades: su origen e importancia}

Las ciudades son centros de aglomeración donde la densidad juega un papel sumamente importante. Son sistemas interconectados compuestos por tres elementos primordiales; infraestructura, operaciones y personas (Cervantes, 2018). Para el arquitecto y planificador urbano francés Alain Bertaud, las ciudades son mercados laborales, es decir, lugares que atraen productores y consumidores y donde los sistemas de transporte son la arteria de dichos territorios. Para él, el futuro de las ciudades depende de cuánta gente esté empleada y cómo se adaptan a las dinámicas del mercado, es decir, sus habilidades para cambiar de empleo. Una definición similar sugiere que las "ciudades son nodos para los flujos globales que apoyan la creación de trabajos de alta calidad y los productos/ingresos económicos" (Rodríguez, 2019: 8). Entre las ciudades que cumplen con estas funciones están principalmente Dubái, Londres, Hong Kong, Nueva York, Singapur y Tokio, porque son integrales y ofrecen todo tipo de oportunidades y oferta de bienes y servicios. La ONU reafirma esta visión al asegurar que "las ciudades son hervideros de ideas, comercio, cultura, ciencia, productividad, desarrollo social y mucho más" (Organización de Naciones Unidas, 2015: 1).

En este sentido, las ciudades se están preparando para que los empleos y las personas estén más cerca y mejor conectados. No en vano, proyectos como "La ciudad de los 15 minutos" ideada en París suenan atractivos, porque permiten que el ciudadano satisfaga sus necesidades en un perímetro acotado que tiene toda la oferta de bienes y servicios que necesita en su diario vivir. Lo anterior da suma importancia a la infraestructura de transporte, que es últimamente la que facilita dicha conexión. Las ciudades inteligentes o smart cities, deben ofrecer condiciones de flexibilidad en materia de planificación y ordenamiento territorial para que las firmas se localicen donde crean más conveniente de acuerdo con los precios y los usos mixtos del suelo. Los usos mixtos ayudan a propiciar mayor dinamismo en las áreas y permiten disminuir los 
desplazamientos, reduciendo así también la contaminación del aire y la congestión vehicular. El uso mixto del suelo propende para que en el suelo coexistan actividades de varios tipos (v.g.: residencia, comercio, trabajo) propiciando así el dinamismo a lo largo del día y la noche. Entre los beneficios de este nuevo enfoque están mayores densidades, menores costos, mayor percepción de seguridad y un modelo de ciudad compacta que "facilita la accesibilidad, reduce costos de infraestructura, consumo de recursos y promueve la integración social" (ONU Habitat, 2017: 1). En contraposición, el modelo mono funcional y de zonificación actual puede inducir a la fragmentación social, asegura ONU Hábitat (ONU Habitat, 2017). El uso del suelo por una sola actividad propicia "bajas densidades, estimula el uso de la movilidad individual y reduce la viabilidad de las redes de transporte público reforzando aún más la exclusión de los menos privilegiados" (ONU Habitat, 2017: párr. 1). Con este enfoque actual las ciudades se vuelven homogéneas y pierden vida en algunos segmentos del día, influyendo así en la calidad de vida que ofrecen al ciudadano.

Aunque las primeras ciudades nacieron en el siglo IV a.C., en los territorios de las antiguas civilizaciones en Asia, el común denominador para el asentamiento definitivo de las poblaciones fue el acceso a los recursos hídricos como ríos y mares. Las ciudades fueron evolucionando gracias al sedentarismo y a las actividades comerciales que fueron dando origen a los mercados y al intercambio. Son territorios de problemas, pero también de oportunidades y soluciones. Estas "se han convertido en espacios privilegiados de realidad, de máxima intensidad colectiva, en los que tiene lugar lo más significativo, para bien y para mal" (Romero, 2018: 32), y las ciudades inteligentes nacieron para dar respuesta a unas necesidades apremiantes y distintas de la era moderna. A lo largo de los años, ha habido características propias de las ciudades que las hacen más atractivas por su gran oferta y porque propician mejores estilos de vida. Así, el mundo ha visto pasar de modelos de ciudades extensas y suburbios a ciudades sostenibles, y ahora inteligentes.

En los últimos años, las ciudades se han desbordado en población, adquiriendo mayor preponderancia a nivel mundial, tanto en las esferas económicas y políticas, así como en la tecnológica. Los alcaldes tienen cada vez más poder e influencia para enfrentar los problemas de forma local' ${ }^{1}$. En el ámbito económico, las ciudades son hubs de una sociedad integrada basada en servicios; políticamente, están en medio de una realineación del poder, con mayor influencia y responsabilidad; y en el tema de tecnología, 
los avances han dado paso a una mayor comprensión de los temas y al control de todo tipo de operaciones y procesos (Dirks y Keeling, 2009: 1). La globalización ha dado pie a una red de ciudades y territorios cada vez más interconectada, generando así mayor competitividad y esfuerzos locales que se traducen en una mayor innovación e ingresos provenientes del sector terciario. En este mismo sentido, el poder se ha reorganizado de forma que los territorios fomentan la participación y eso, a su vez, brinda mayor legitimidad a los gobernantes para la toma de decisiones. Las ciudades son espacios de gran participación y cambio que influyen a nivel regional e incluso mundial. Finalmente, en el ámbito tecnológico ha sido más fácil percibir esta transformación porque los cambios son más tangibles y visibles. La ciudad inteligente es hoy la meta de todas aquellas que guardan la esperanza de formas de vida más fáciles.

\section{Ciudades inteligentes - Smart Cities}

Este tipo de ciudades nacieron aproximadamente hacia el 2008 con la crisis financiera, "encontrando las empresas de tecnología un nuevo nicho de mercado en el ámbito de la gestión local" (Romero, 2018: 32) y entendiendo cómo la "relación entre gobernanza y tecnología se convierte en una fórmula de gestión pública" (Romero, 2018: 32). El Gráfico 1 revela algunos de los componentes más importantes que debe tener una ciudad inteligente. En él se exponen conceptos en todos los sectores; medio ambiente, provisión y gestión de los servicios públicos, vivienda, salud, seguridad, educación y datos, entre otros. Si bien cada uno de esos términos es de gran relevancia, la ciudad como un todo funciona cuando se integran de manera ordenada y sincronizada dichos componentes. Eso es precisamente una ciudad, un ecosistema compuesto por pequeños subsistemas que se entrelazan entre sí para formar redes que permitan que el territorio funcione. 


\section{Gráfico 1. Componentes de una Ciudad Inteligente}

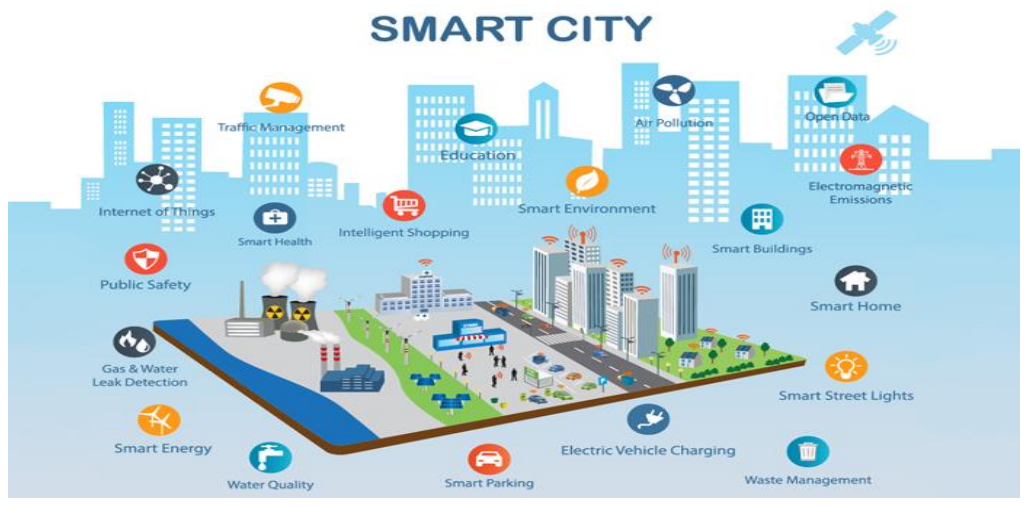

Fuente: Mateu, 2018

Si bien a primera vista pareciera que las ciudades inteligentes solo buscan hacer un mayor uso de las tecnologías, estas se orientan a ser inteligentes en un sentido más amplio. Dicho sentido se extiende a hacer un uso más eficiente de los recursos, a aprovecharlos de forma que se reduzcan las emisiones de $\mathrm{CO} 2$ y el consumo energético, todo ayudado por las nuevas herramientas tecnológicas. Aunque en este tipo de conceptos no hay una sola definición, a continuación, se presentan algunas descritas por organizaciones multilaterales y empresas de servicios tecnológicos que están a la vanguardia del desarrollo económico y social sostenible.

Para el Banco Interamericano de Desarrollo (BID), "una ciudad inteligente pone a las personas en el centro del desarrollo, incorpora las Tecnologías de la Información y la Comunicación (TIC) en la gestión urbana, y utiliza estos elementos como herramientas para estimular el diseño de un gobierno que incluye la planificación colaborativa y la participación ciudadana" (Bouskela y Elnir, 2018: párr. 4). De esta forma, se logra un desarrollo integrado y sostenible y las ciudades se vuelven más innovadoras, competitivas, atractivas y resilientes. Según el BID, algunas de las características más importantes de una ciudad inteligente son las siguientes. En primer lugar, está la sostenibilidad, cuyo fin se logra mediante la interacción de la administración pública y el sector privado, y en el cual su objetivo principal es hacer uso eficiente y consciente de los recursos, reduciendo costos y optimizando el consumo. La inclusión y la transparencia ayudan, por su parte, a que la comunicación fluya hacia los ciudadanos mediante procesos de datos abiertos que permiten la 
participación de estos en proyectos políticos y sociales de interés común. Adicionalmente, una ciudad inteligente debe garantizar la generación de riqueza mediante empleos de calidad, innovadores y competitivos. Finalmente, los ciudadanos son el núcleo de la ciudad y, por ende, se busca satisfacer sus necesidades de manera integral y rápida (Ospina, 2018).

Para la multinacional de tecnología IBM, una ciudad inteligente propende por el "equilibrio inteligente que se encuentra entre la tecnología, como base, y el ambicioso objetivo del desarrollo sostenible y la mejora de las condiciones de vida urbana" (Sajhau, 2017: 52). Según la empresa tecnológica, hay tres elementos que definen una ciudad de este tipo: una plataforma de datos que recoge y agrega información para la toma de decisiones; disponibilidad de datos a nivel regional y local por medio de políticas de datos abiertos; y la participación ciudadana en todas las actividades y procesos (Sajhau, 2017: 53).

Por su parte, para la compañía de automóviles Toyota, una ciudad inteligente es una ciudad entrelazada (woven), es decir, "un modelo de urbe totalmente conectada, sostenible, con vehículos autónomos, basada en energía solar y en las pilas de combustible de hidrógeno" (Esmarcity, 2020: párr. 1). La multinacional avanza con rapidez en un modelo de ciudad que será explicada en detalle más adelante.

Si bien hay elementos importantes que no están contenidos en estas definiciones, quizás uno que hay en común y que, a su vez es de gran valor, es la gobernanza y la importancia de los ciudadanos como actores fundamentales de este modelo. Para lograr un verdadero desarrollo sostenible, es necesario dedicarle tiempo a la planeación de las actividades, así como a su seguimiento, control y posterior evaluación. La gobernanza de las ciudades debe ser pensada de forma sostenible, maximizando las oportunidades económicas y minimizando los daños ambientales (Ospina, 2018). En este contexto, el ciudadano entra a jugar un rol importante en la construcción de ciudad para legitimar al gobierno y satisfacer sus necesidades de la mejor manera.

En síntesis, una ciudad inteligente es aquella que es más eficiente y sostenible mediante el uso de infraestructuras limpias como paneles solares, medios de transporte eléctricos, etc. (Sostenibilidad, s.f.) y donde el ciudadano ejerce un rol importante en cada una de sus etapas. Las ciudades inteligentes generan impactos positivos en los ciudadanos porque, además de afectar la esfera digital, "habilita nuevos enfoques de desarrollo que 
permiten cerrar brechas socioeconómicas en diferentes dimensiones" (Universidad del Rosario, 2020: 1). Aunque no hay un modelo único que pueda copiar y pegarse en todos los territorios, sí hay elementos comunes que pueden adaptarse en una u otra ciudad con el fin de aprovechar el contexto económico, ambiental y social de cada una. Comprender qué ofrece una ciudad, qué necesidades tiene, con qué fortalezas y debilidades cuenta, es el primer paso para avanzar hacia la construcción de una ciudad inteligente.

\section{Economía circular}

El sistema de producción y consumo actual está basado en un modelo lineal enfocado en la extracción, transformación y desecho de los recursos (Stagno, 2020). Desde la Revolución Industrial, a finales del siglo XIX, el mundo adoptó el sistema capitalista que a su vez llevó al modelo de producción y consumo actual. Dicho modelo refleja la linealidad de los procesos en el cual un recurso se usa para producir un bien que a su vez se utiliza hasta que cumple su vida útil y luego se desecha. A pesar de que dicho modelo se ha mantenido por mucho tiempo, hoy en día no es sostenible porque la presión sobre los recursos se ha exacerbado de forma inmensurable, el consumo se ha desbordado y los residuos se han multiplicado. El BID asegura que "las ciudades son responsables del consumo de $75 \%$ de los recursos naturales, $66 \%$ de la energía producida y $54 \%$ de los materiales del mundo, y se espera que para 2050 consuman el 80\% de los alimentos disponibles" (Stagno, 2020: párr. 2). Por consiguiente, los recursos se están agotando, los desperdicios han aumentado y el impacto sobre el medio ambiente es irreversible. De hecho, estimaciones del World Economic Forum y Accenture Strategy sugieren que "actualmente se necesita la capacidad regenerativa equivalente a 1.7 planetas Tierra para reponer los recursos consumidos y absorber la contaminación generada por nuestros sistemas productivos. Para el 2050 se necesitará el equivalente a 3 planetas" (World Economic Forum y Accenture Strategy, 2019: 7).

La siguiente imagen expone la diferencia entre ambos modelos. La parte superior revela cómo el proceso lineal termina cuando los bienes y recursos parecen no servir más y se convierten en desechos. Por su parte, la imagen inferior refleja el proceso circular de los bienes y recursos de la economía, en la cual se reduce al máximo su desperdicio y se aprovecha todo su potencial, incluso cuando estos parecen no servir más. 


\section{Gráfico 2. Modelo de economía lineal vs. circular}

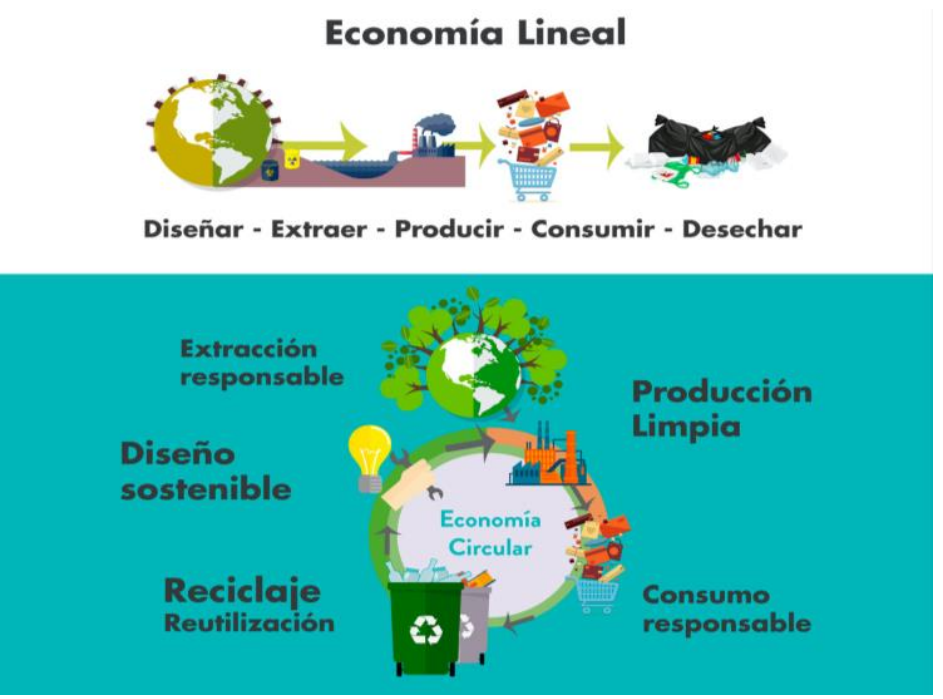

Fuente: Sánchez, 2019

El modelo propuesto en el que una ciudad inteligente debe basarse es, entonces, el de la economía circular. Como su nombre bien lo indica, lo que está detrás de este concepto es alargar la vida útil de los recursos y los bienes, lo que "implica mantener materiales en circulación de manera indefinida, obteniendo el mayor valor posible antes y después de su desecho" (Stagno, 2020: párr. 4) y, de la misma forma, minimizar los desperdicios durante todas las etapas de producción de un bien. El modelo se basa "en disciplinas como la ecología industrial, que tiene como metáfora la búsqueda de sistemas de producción y consumo cuyo funcionamiento se asemeje al de los ecosistemas naturales con baja perturbación" (Rodríguez, 2019: 53). En resumidas cuentas, la economía circular busca mantener activos los bienes y recursos en vez de reemplazarlos, evitando así el modelo consumista que predomina en el mundo y propiciando modelos de consumo colaborativo y de intercambio donde "en lugar de deshacerse de los materiales como residuo, se valoran como insumos en unos próximos ciclos de producción” (Rodríguez, 2019: 196).

El modelo circular se basa en las $7 \mathrm{R}$; repensar, rediseñar, reducir, reusar, reparar, reciclar y recuperar. Dichas acciones están encaminadas a minimizar la generación de residuos y la contaminación, así como apoyar plataformas de economía colaborativa y reciclaje. El siguiente gráfico 
muestra cómo funciona el modelo y sus principales componentes para producción y uso de bienes y recursos.

\section{Gráfico 3. Principios del Modelo de Economía Circular}

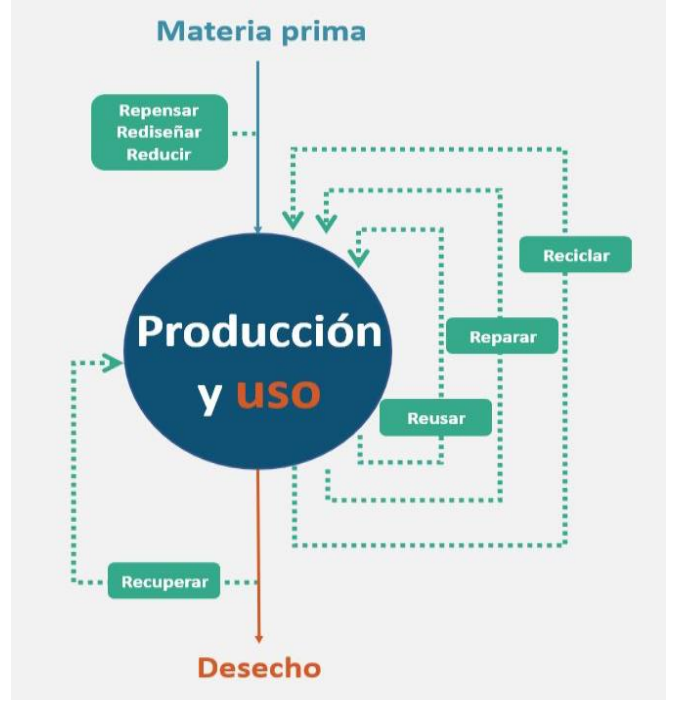

Fuente: Stagno, 2020

Según un reporte del Foro Económico Mundial y la Ellen MacArthur Foundation, se estima que si el mundo adoptara los procesos de la economía circular se "generaría un ahorro de materiales de 1 billón de dólares al año; cifra equivalente al PIB de naciones como Holanda o Indonesia" (Stagno, 2020: párr. 6). Sumado a eso, está el hecho de todo lo que se ahorraría el mundo en materia de desechos sólidos que serían de un valor aproximado de 20 millones de toneladas métricas al año (Stagno, 2020), así como en reducción de gases efecto invernadero (48\%) a 2030 y reducción de costos de movilidad (50\%), vivienda (25\%-35\%) y alimentos (25\%-40\%), específicamente para el caso de Europa.

\section{Ciudades nocturnas y alcaldes de la noche}

Las ciudades de hoy son igualmente activas y dinámicas tanto en el día como en la noche. Aunque esta característica no se evidencia en todo el mundo, cada vez son más las ciudades cuya economía nocturna adquiere mayor importancia. "Cada ciudad tiene una historia y una vocación nocturna distinta, y su estrategia para manejarla debe ser igualmente única, 
basada en las necesidades y expectativas de sus ciudadanos" (Seijas, 2018: 3). Entender la vocación de cada ciudad es la primera tarea para implementar este tipo de estrategias y que perduren en el tiempo. De igual forma, y en especial en América Latina, hay retos y obstáculos como el "ruido, la violencia, la desigualdad y la informalidad" (Seijas, 2018: 3). La idea de la nocturnidad urbana puede tener una connotación negativa asociada a la rumba y el trago, sin embargo, sus beneficios pueden ser mayores y generar externalidades positivas para el interés colectivo (Seijas, 2018). No en vano, varias ciudades alrededor del mundo están adoptando estrategias para aumentar la intensidad de las actividades nocturnas ahora que reactivan sus economías durante la pandemia del COVID 19, generando así distintos turnos de trabajo que soportan el distanciamiento social y los principios de no aglomeración para reducir los contagios. La noche es, entonces, una excelente opción que favorece el distanciamiento social y que puede reducir costos a la vez que ofrece ventajas en otros campos como ya se ha mencionado.

La noche es sinónimo de descanso y cierre de jornada laboral para unos, pero para otros significa la continuación de sus deberes. Es el caso de médicos, sector seguridad, y logística, por ejemplo. La actividad nocturna se suele considerar entre 6:00 de la noche y 6:00 de la mañana, lo que refleja así el periodo comprendido entre el atardecer y el amanecer. Hoy, más que nunca, con la emergencia generada por el COVID 19, se percibe cómo las profesiones que trabajan en la primera línea y en las noches, se han convertido en las más importantes. Por otro lado, están las actividades nocturnas que hoy no generan ingresos y que podrían aumentar su relevancia una vez se logre avanzar al final de la pandemia. Es el caso de los bares y discotecas, de los cines y los restaurantes. Solo un cambio de paradigma permitirá que la actividad nocturna logre consolidarse con una mayor participación dentro de la economía local y nacional.

El concepto de la ciudad nocturna radica que haya actividad las 24 horas del día. Es una propuesta de convivencia y valor económico en otro espacio de la ciudad según la experta venezolana Andreina Seijas. Entre las ventajas de este modelo está el aumento de turismo y empleo, y la apropiación de la ciudad por parte de sus ciudadanos, que puede ayudar a mejorar la seguridad al mantener las calles llenas de vida. La apertura de las ciudades a la noche genera una fuente de ingresos adicionales, además de ayudar a revitalizar el espacio público y diversificar la oferta de servicios. En Reino Unido, por ejemplo, se estima que la noche da empleo a 1.3 millones 
de personas en actividades que involucran servicios de todo tipo que incluyen bibliotecas, gimnasios y farmacias, entre otros. Por otro lado, en Nueva York, la economía nocturna en 2019 generó ingresos por USD \$35,1 billones y cerca de USD $\$ 700$ millones por concepto de impuestos locales (Masud, 2019).

Dentro de las condiciones que debe tener una ciudad para funcionar todo el día está la infraestructura (hardware), incluyendo los sistemas de transporte y alumbrado público. Adicionalmente, están las normas y regulaciones (software) para que los servicios funcionen de manera adecuada. Otro componente importante es la apropiación de los espacios por parte de los ciudadanos. Como afirmaba la activista Jane Jacobs, entre más ojos en la calle haya, más seguridad habrá. Para que este modelo funcione de forma eficiente es necesario que haya un agente/alcalde/gerente de la noche. Ámsterdam, en Holanda, es pionera en asignar un alcalde nocturno a su ciudad. Esta persona está encargada de mapear los establecimientos y regular la actividad de la noche, además de "promover una economía nocturna de la ciudad que conduzca a la generación de empleo mediante actividades sociales, culturales, recreativas, comerciales, deportivas y gastronómicas, porque una calle o una zona llena de gente se percibe como un sitio seguro y pleno de vida" (Benítez, 2019: párr. 8).

Para que una ciudad realmente pueda ofrecer servicio nocturno, es necesario la participación y el activismo de todos los grupos poblacionales y profesionales. Por un lado, están todos los asistentes y quienes disfrutan de la oferta cultural nocturna. Por otro lado, están quienes ofrecen dichos servicios, los que trabajan durante la noche. Finalmente, están todos los que administran la actividad nocturna que incluye personal de seguridad y gobierno, principalmente. Con tantos sectores involucrados se hace totalmente necesario planificar y gestionar estas actividades de forma regular y organizada, para garantizar la seguridad y coordinar dichos actores en pro de la calidad de vida de los habitantes. En los últimos años, ha quedado demostrado que los proyectos funcionan cuando tienen la participación de varios sectores y actores, pero, sobre todo, cuando todos ellos se coordinan. En este sentido, las asociaciones público-privadas (APP) deben fortalecerse por medio de canales de comunicación claros y fluidos. Aunque las APP son mundialmente reconocidas, la academia se suma a esta alianza para fortalecer a las comunidades y los procesos que se llevan a cabo en las ciudades. 
Otro de los aspectos que definen una ciudad nocturna, e inteligente como se verá más adelante, es el uso de herramientas tecnológicas y los datos. Estos últimos permiten conocer en tiempo real el comportamiento de la ciudad, los puntos neurálgicos y las necesidades de los ciudadanos. Solamente teniendo información actualizada y de primera mano, se puede hacer seguimiento y control a las actividades de la noche.

Otra razón para promover las actividades nocturnas es pensar en quienes todo el día trabajan y tienen horarios de salida al final del día o ya en la noche, cuando el comercio y muchos servicios han cerrado su atención al público. Algunas ciudades adelantan actividades esporádicas en la noche como ciclovías nocturnas, la noche de los museos, rebajas en los establecimientos comerciales, entre otras, pero en general la noche sigue siendo vista como un espacio temporal en que la ciudad duerme y todo se apaga (Seijas, 2018). Una ciudad $24 / 7$ es potencial de oportunidades para todos. Los siguientes son tres ejemplos de ciudades que han tenido éxito en este tema en los últimos años.

\section{a. Ámsterdam}

Esta ciudad holandesa "se convirtió en la ciudad líder en iniciativas para proteger y maximizar la oferta nocturna" (Seijas y Sound Diplomacy, 2018: 41) equilibrando así las necesidades de sus propios habitantes con los de miles de turistas que la visitan anualmente. Para garantizar la seguridad de sus calles, el alcalde nocturno puso a disposición de sus habitantes "una aplicación móvil para que los residentes pudieran reportar perturbaciones [...]. La app conecta al usuario con el oficial más cercano, a fin de dar respuesta rápida a la denuncia" (Seijas y Sound Diplomacy, 2018: 42).

\section{b. Montreal}

En América, "Montreal es la campeona de la economía nocturna. Incluso durante el invierno, la ciudad se caracteriza por una gran vida cultural y gastronómica tanto de día como de noche. Los negocios de los distritos más movidos y con mayor oferta tienen la opción de permanecer abiertos durante toda la noche como una medida para dinamizar la economía local" (Seijas, 2015a: párr. 8). 


\section{c. Buenos Aires}

La capital argentina es otra de las ciudades pioneras en cuanto a este tema en América del Sur. Desde hace unos años, la ciudad organiza "La Noche de los Museos", una ocasión anual en la que se reúnen miles de personas para celebrar el arte y la cultura de la ciudad (Seijas, 2015a).

\section{Gobernanza y participación}

La ciudad inteligente toma como base en el tema de gobernanza el concepto de "ojos en la calle" que planteó la activista y escritora Jane Jacobs. Adicionalmente, su modelo de ciudad era concebido con densidades medias, una diversidad de personas, actividades y sectores económicos infinita, plantas bajas activas, usos mixtos y espacios públicos para el ciudadano (Janka, 2016). Es una ciudad más humanizada, donde el habitante tiene derechos y responsabilidades. Entre sus responsabilidades en la calle y el espacio público, el ciudadano debe observar y reportar cualquier anomalía, se cuidan entre todos y así aumenta la percepción de seguridad. En las ciudades inteligentes se crean comunidades y lazos fuertes entre los vecinos con el fin de hacerla más dinámica, activa, segura y participativa. La relación es que, si hay más gente en la calle, hay más ojos y más control, lo que reduce la inseguridad. Un espacio más seguro incentiva a una mayor participación ciudadana y al uso efectivo de la ciudad, generando un ciclo virtuoso que mejora la calidad de vida de los habitantes. Este concepto se suma al apartado anterior de ciudades nocturnas, puesto que refuerza la importancia de que haya actividad durante la noche y que, además, sean espacios seguros donde los mismos ciudadanos se vigilen y ejerzan control entre ellos. Se producen, entonces, ciudades incluyentes, seguras y dinámicas, ciudades inteligentes al final.

De las características más relevantes que tienen que considerarse a la hora de hablar de ciudades inteligentes es la gobernanza. Dicho concepto incluye no solo la parte proveniente de la administración pública sino desde los ciudadanos mismos. La ciudad inteligente de hoy, inter e hiper conectada, requiere un modelo de datos abiertos que permita a los ciudadanos informarse en tiempo real con cifras y datos oficiales. Afirma Enrique V. Iglesias, ex presidente del BID que "no es suficiente con tener ciudades inteligentes, también hace falta tener ciudadanos inteligentes" (Bouskela et al, 2016: 6). Un ciudadano inteligente es aquel que, contando con información oportuna y confiable, participa en la construcción de ciudad. El ciudadano que se requiere hoy es un sujeto consciente de lo que 
ocurre en su entorno, es un individuo que propicia un sentido de pertenencia fuerte tanto con su comunidad como con su territorio. Hoy en día, la información fluye en todas las direcciones y el acceso a las herramientas tecnológicas es más grande que nunca, por eso la importancia de los datos y que la ciudad inteligente ponga al ser humano como eje de su ecosistema. Los habitantes de las ciudades votan hoy con sus pies, esto significa que, si una ciudad no les provee lo que necesitan, se marchan a otra.

Desde hace unos pocos años, los ciudadanos empezaron a ser sujeto de deberes y no solo de derechos. Con ello, empezaron a interesarse y a inmiscuirse en los ámbitos social y político de sus economías, exigiendo que los gobiernos de turno cumplieran a cabalidad con sus planes y, sobre todo, con el mejoramiento de la calidad de vida de los habitantes de forma equitativa. Hoy se habla con mayor preponderancia de democracia participativa, aquella en la cual los ciudadanos no solo votan, sino que participan en las distintas esferas; aquella en la que personajes de toda clase social, origen, religión y género tienen representación en los gobiernos locales y nacionales.

La descentralización política ha sido un ideal en muchas naciones, en especial, porque permite que las ciudades se desarrollen localmente y tengan mayor control de sus acciones. Adicionalmente, permite que los ciudadanos sean más activos porque se sienten más cercanos a sus gobernantes y la información fluye en ambas direcciones. Cuando los ciudadanos se empoderan porque tienen información de primera mano, la percepción de transparencia y confianza ciudadana en los gobiernos aumenta, lo que se traduce en una mayor legitimidad y gobernanza pública. Los gobiernos deben bajar al terreno local con un sentido de voluntad política real que invite al ciudadano a interesarse por su ciudad. Deben también propender por una fuerte cultura de innovación que vaya evolucionando y adaptándose a las dinámicas ciudadanas y territoriales que van apareciendo con frecuencia. Uno de los grandes retos al interior de los gobiernos es que los funcionarios no se comunican, por eso deben organizarse internamente antes de llevar a cabo procesos externos de mejora ciudadana.

De igual forma, este empoderamiento ha dado lugar a un sinnúmero de organizaciones que inician desde los ciudadanos mismos (grassroots movements) y desde las cuales se han originado proyectos integrales que repercuten en mayores beneficios. No en vano, estos proyectos tienen, al 
final, más eco y éxito en las ciudades, porque el desarrollo económico local permite que los habitantes reconozcan sus necesidades, expongan sus argumentos y planteen soluciones sólidas a mediano y largo plazo. Uno de los ejemplos que refleja lo anterior es el urbanismo táctico. La implementación de esta herramienta inicia cuando los ciudadanos se hacen notar y ponen de manifiesto sus necesidades para intervenir un espacio específico. El diagnóstico acertado de la comunidad y el territorio es lo que lleva a mejorar estos espacios por medio de la pintura, la señalización y el mobiliario urbano adecuado, entre otras estrategias.

\section{Tecnología}

Hay que reconocer que las ciudades inteligentes tienen un componente importante que proviene de las tecnologías de la información y la comunicación. Los datos, las cifras y los números, son hoy más que nunca referencias obligatorias para monitorear todas las actividades que ocurren en una ciudad. Sin embargo, el rasgo más importante dentro de este tema es el de la interoperabilidad de los sistemas. Una ciudad inteligente tiene muchos frentes, muchos datos, mucha información proveniente de múltiples fuentes de información. En este sentido, se hace totalmente necesario conectar e integrar dichos datos en una plataforma y explotarlos para el bien común (Garcia, 2018: 1). Existe la idea de que "la vida en la ciudad es el resultado de interacciones complejas" (Mehmet, 2020: párr. 3), y con la disponibilidad y el uso de datos e información de alta calidad se hace más fácil comprender dichas relaciones.

La tecnología es un medio mas no un fin para el desarrollo y la implementación de las ciudades inteligentes (Sajhau, 2017: 1). Si bien las smart cities se entienden a partir del uso de herramientas tecnológicas, dicho componente no es exclusivo, no es su fin, sino más bien el camino para desarrollarlas. La ciencia de datos, su análisis y la computación de estos ayudan a crear diagnósticos y predecir eventos debido a una mejora en la información. Con esto, los tomadores de decisión cuentan con más y mejores instrumentos para tomar decisiones acertadas. Es este aspecto el que le da el verdadero sentido al concepto de ciudades inteligentes, el buen uso de los datos y la tecnología para lograr soluciones sostenibles a las necesidades de los ciudadanos. Por su parte, IBM asegura en el mismo sentido que "nuestro papel (el de la empresa) no es tomar el lugar de los tomadores de decisiones, sino más bien informarles y ayudarlos a tomar rápidamente la decisión correcta" (Sajhau, 2017: 56). 
La política de datos abiertos va en congruencia con los modelos de $e$ governance y transparencia de los gobiernos, que no solo deben rendir información al ciudadano sino dotarlo de información clara y precisa que pueda utilizar en su día a día. En suma "lo que hará inteligente a una ciudad no será el número de sensores o apps que tenga, sino cómo y para qué utilizará la ciudad esa tecnología" (Garcia, 2018: 2). McKinsey Global Institute asegura que las soluciones digitales pueden mejorar algunos indicadores de calidad de vida en un $30 \%$, en especial porque ayudan a la prevención, como es el caso de los sensores de los hogares o los equipos electrónicos ubicados en las calles.

Con el auge de las ciudades inteligentes, ha aumentado también la participación del sector privado, en especial en los temas de tecnología. En 2009, IBM fue pionera en desarrollar un modelo de ciudad inteligente cuya misión es crear un "enfoque integral para ayudar a las ciudades a funcionar de manera más eficiente, ahorrar dinero y recursos, y mejorar la calidad de vida de los ciudadanos" (Harnish, 2016). La compañía promete transformar la forma en que las personas viven ahora y en el futuro, y para ello considera que los tres elementos básicos son las personas, los beneficios y el planeta. Ellos creen que el uso de la tecnología puede aliviar y dar respuesta a las necesidades de los ciudadanos y para ello han desarrollado softwares eficientes y de tecnología de punta para varios sectores y temas.

Las fuentes de información son la materia prima para el análisis de datos, lo cual permite realizar diagnósticos y conocer tendencias para proponer soluciones a largo plazo. Aunque hoy la gente sienta que vive en constante vigilancia, la realidad es que cada segundo se producen billones de datos que, al integrarse, producen resultados que cambian la forma en que la administración pública y el sector privado atienden las solicitudes ciudadanas (Harnish, 2016). En este sentido, la infraestructura digital juega hoy un rol crucial en las ciudades. IBM señala que la importancia de los datos no es su cantidad sino su calidad y utilidad posterior. El mayor incentivo que tiene la empresa es que asegura que a mayor crecimiento demográfico, mayores serán las demandas de los habitantes y que sin datos reales, presupuestos limitados, recursos insuficientes e infraestructuras desgastadas, será muy difícil atenderlas (Harnish, 2016). Adicionalmente, y como resultado, la presión hacia el medio ambiente se disparará, por eso se hace necesario conocer en tiempo real los recursos con los que se cuentan, y cómo todo está interconectado, lo que se haga de un lado afectará lo que se haga en otro. 
Por otro lado, la multinacional Toyota, comenzará a construir su ciudad entrelazada (woven) en Japón en 2021. Se ha denominado a este tipo de ciudad entrelazada ya que pretende "potenciar la conexión interpersonal y entrelazar el entorno urbano con la naturaleza" (Esmarcity, 2020: párr. 13). El objetivo es hacer de una ciudad real un laboratorio para poner a prueba la tecnología teniendo como sustento los siguientes 16 pilares: comunidad, movilidad personal, vehículos autónomos, robótica, hogares inteligentes, inteligencia artificial, movilidad como servicio, viviendas asistidas multigeneracionales, naturaleza, salud, estructuras de energía de hidrógeno, investigación académica, colaboración entre industrias, y construcción y producción inteligente (Esmarcity, 2020). Aunque inicialmente la ciudad albergará a 2.000 personas relacionadas con la multinacional japonesa, la ciudad podrá crecer conforme a su evolución. Entre los rasgos que definirán la ciudad está el de dividir las vías para tres categorías diferenciadas: vehículos autónomos, peatones y un parque/alameda. De igual forma, todas las edificaciones serán construidas con madera neutral en emisiones de carbono, y la estructura subterránea albergará un "almacén de energía de hidrógeno, sistemas de filtración de agua, así como una red de entrega a domicilio totalmente autónoma que se conecta con los inmuebles" (Esmarcity, 2020: párr. 9). Por su parte, los techos contarán con paneles fotovoltaicos para generar energía solar. Al interior de los hogares todo estará basado en inteligencia artificial y sensores. Sin embargo, antes de poner en marcha la construcción de esta ciudad, el plan es diseñarla y probarla en el plano digital.

La llegada del Internet de los Objetos/Cosas (IO), gracias a las innovaciones en la tecnología de sensores más barato, grandes volúmenes de datos y análisis predictivo, está haciendo posible hacer frente a todo tipo de problemas urbanos (Secure Week, 2017). No es casualidad que hoy en día se estén desarrollando tantas aplicaciones, no solo en materia social (Facebook, Instagram, LinkedIn), sino aplicaciones con impactos reales que repercuten en el bienestar de la ciudadanía. Ejemplo de ellos son las aplicaciones de movilidad o "ride sharing" como UBER o Cabify, o WAZE, que en tiempo real anuncia donde hay congestión en las calles y las rutas más eficientes para llegar de un lugar a otro. En el sector de hostelería y turismo, AirBnb se cataloga como la más popular. El rasgo común de estas herramientas es facilitarle al consumidor/ciudadano la búsqueda de información, y que pueda llevar su estilo de vida de una forma rápida y sencilla. 
En otros sectores, la tecnología está ayudando a sostener las ciudades inteligentes de las siguientes formas: mediante vehículos eléctricos con sus respectivos puntos de recargas, sistemas de bicicletas compartidas y las scooters eléctricas, las tarjetas con sensores que pueden estar en los celulares y que sirven para pagar y montar en transporte público, entre otros ejemplos. Todo parece estar al alcance de los ciudadanos con solo un clic. Como se mencionó anteriormente, la inteligencia en los edificios y hogares se percibe en un consumo menor de energía y en sistemas de optimización de calefacción, así como en los jardines y la vegetación de sus edificios que ayudan no solo con el aspecto estético, sino con el aislamiento y la recolección de aguas lluvia también. En países europeos, por ejemplo, hay un excelente monitoreo de los niveles de basura en los contenedores ubicados en las aceras en tiempo real para optimizar las rutas de recolección.

Con la emergencia mundial actual, la tecnología seguirá imponiéndose como una opción segura y válida que ayude a evitar el contacto entre personas y objetos, mejorando así los servicios ciudadanos y generando confianza entre quienes habitan las ciudades al facilitar los estilos de vida. No en vano, "las políticas entorno a las TIC puedan acelerar y consolidar el progreso socioeconómico” (Universidad del Rosario, 2020: 4).

A pesar de las ventajas visibles que ofrece la tecnología, hay quienes se oponen a ella debido a dos factores, en especial, a la sobrevigilancia de los gobiernos y a la inequidad que genera el acceso limitado a la tecnología. Si bien la tecnología pone a disposición una gran cantidad de información y datos abiertos, para ello necesita auscultar en gran parte de la vida de sus ciudadanos. Lo anterior hace que, en ocasiones, la línea divisoria entre los ámbitos público y privado se borre un poco. El mayor riesgo es que hackeen los sistemas, se socialicen los datos de los habitantes y los utilicen en su contra. Por lo anterior, tanto el gobierno como los privados deben generar sistemas de alta calidad que eviten la filtración de datos. Otro de los retos es la brecha digital, no solo a nivel mundial, sino también entre las áreas rurales y urbanas. La brecha digital se suma hoy a la ya existente e indeseada brecha socioeconómica por la que tanto luchan los países. Sin embargo, como advierte Sokwoo Rhee, pionero en la creación de tecnologías, modelos como el wifi público, pueden ayudar a mitigar este obstáculo al ofrecer acceso gratuito a Internet en espacios públicos (Naím, 2020). 


\section{América Latina}

La región de América Latina es una de las áreas con mayor concentración urbana del planeta, por consiguiente, los retos a los que se enfrenta también corresponden a esa escala, encontrándose con problemas comunes para todas las ciudades del territorio. En general, las ciudades de la región exhiben mayores rezagos en los aspectos de economía, vinculados con las estrategias de desarrollo económico e innovación de las ciudades, de escasez de capital humano, de baja capacidad respecto a la proyección internacional, y de menor acceso a la tecnología entre sus ciudadanos (Ospina, 2018). Por ejemplo, las ciudades latinoamericanas están un poco rezagadas con respecto a sus pares europeas o norteamericanas en materia de economía circular y, en su mayoría, se concentran en el reciclaje como forma de mitigar los efectos negativos en el medio ambiente. Aún hace falta tener en cuenta las otras seis $\mathrm{R}$.

Por otro lado, si bien en América Latina se cuenta con numerosas aplicaciones tecnológicas que facilitan el día a día de los ciudadanos, todavía "se presentan fallas que retrasan la entrega de datos de manera inmediata" (Metro Cuadrado, 2020: párr. 7). En este sentido, se puede afirmar que todavía falta un sistema que integre y procese los datos y la información que se obtiene de las distintas herramientas puestas a disposición del ciudadano. Falta el aspecto holístico que es necesario en una ciudad inteligente, esa interoperabilidad de la información y la integración con el ciudadano. Desde la Universidad de Navarra, que desde hace un par de años estudia este tipo de ciudades, se afirma que "aún no existe una política gubernamental clara sobre las ciudades inteligentes" (Metro Cuadrado, 2020: párr. 10) en estas latitudes. Sin embargo, vale la pena mencionar el enfoque que los gobiernos actuales están aplicando en cuanto a temas de innovación, emprendimiento y economía naranja, lo cual ha permitido un mayor número de aplicaciones e ideas que se encuban en América Latina.

Smart City es el modelo al cual están migrando las ciudades que quieren crecer sosteniblemente para ofrecer a sus habitantes los beneficios que traen los avances tecnológicos del siglo XXI. En América Latina, aunque en la actualidad sean pocas las ciudades inteligentes, se puede evidenciar una verdadera mejora en cuanto a la institucionalidad, la cooperación público-privada y los requisitos tecnológicos necesarios para lograr ciudades más inteligentes en los próximos años. De parte de los ciudadanos, la capacitación y concientización de la necesidad de crear un desarrollo sostenible que aproveche los avances tecnológicos también es parte de los 
desafíos que conlleva la transformación hacia ciudades inteligentes (Ospina, 2018).

\section{Conclusiones}

En primer lugar, es innegable comprender las ventajas que ofrecen los modelos de ciudades inteligentes no solo por la cantidad de su oferta, sino por sus impactos. Sin embargo, aunque el modelo plantee ciertos elementos como requisito para desarrollar este tipo de ciudad, no hay que olvidar que cada ciudad es diferente y única. Cada ciudad necesita, entonces, su propio diagnóstico para encontrar sus ventajas y fortalezas, así como sus debilidades y amenazas. Una vez que se entiende en qué momento se encuentra la ciudad y qué necesita, se plantea una visión de mediano y largo plazo que apunte a una serie de metas tangibles para llevarla por la senda adecuada. La ciudad del futuro satisface las necesidades de la población, pero solo conociendo de antemano sus requerimientos puede hacerlo de forma eficiente. Las necesidades básicas, el bienestar integral y la sostenibilidad deben ser la prioridad.

Las ciudades inteligentes no se limitan solo a la creación de aplicaciones, sino a la forma en que se gestionan las ciudades teniendo como base una visión de ciudad a largo plazo. La ciudad "necesita ser el sujeto de una ambición colectiva transformando la relación entre los varios jugadores" (Sajhau, 2017: 57), como se ha expuesto a lo largo del documento. Para lograr lo anterior, se hace totalmente necesario que exista una continuidad en los proyectos, es decir, que haya no solo una visión de largo plazo compartida, sino que los gobiernos de turno se comprometan con el tema. Específicamente, "el rol de los gobiernos locales es el de permitir el crecimiento sostenible creando interacciones con el mercado, construyendo la infraestructura mínima viable, y compartiendo las mejores prácticas con otras regiones para facilitar la adopción mientras se garantiza el beneficio social" (UN-Habitat, 2020: 101). Por consiguiente, la "política pública debe encargarse de proveer las condiciones básicas para generar desarrollo en paralelo con la transformación digital" (Universidad del Rosario, 2020: 4) y así sentar las bases para que las ciudades inteligentes sean una realidad.

El mayor atractivo del concepto de smart cities quizás sea su multidimensionalidad, ya que persigue mejorar la calidad de vida en las ciudades mediante la aplicación de nuevas tecnologías y métodos de gestión en diversas áreas desde las infraestructuras, medio ambiente, energía y 
movilidad, hasta la facilitación de la actividad económica (Mateu, 2018). Así como los mayores problemas de las ciudades tienen aspectos en varios frentes, así deben ser tratadas sus soluciones, de manera holística e integral. Por lo anterior, la ciudad inteligente deberá "proveer ecosistemas vibrantes, fluidos, flexibles" (Rodríguez, 2019: 12) que propicien espacios para vivir, trabajar y recrearse. En resumen, una ciudad inteligente es aquella que tiene un tamaño intermedio que permite el diseño a escala humana y en el cual la micro movilidad prima para que los mercados laborales funcionen dinámica y eficientemente.

Aún faltan diez años para lograr cumplir las metas planteadas en los ODS, y aunque ahora todo esté frenado por el COVID 19, las ciudades seguirán siendo fundamentales a la hora de dar respuesta a las necesidades más apremiantes de los ciudadanos. La interacción de todos los actores que tienen un papel crucial en el desarrollo social, económico y político de las ciudades será clave para salir de la crisis que deje la pandemia. El contexto actual es propicio para apoyar el modelo que sugiere la ciudad inteligente respecto a la economía colaborativa, no solo en el tema de las aplicaciones tecnológicas, sino en el ámbito de la economía nocturna y la economía circular que puede aplicarse en los distintos sectores, como se mencionó anteriormente. Hay que rescatar, además, que muchos proyectos y estrategias digitales alrededor del mundo han tenido en estos últimos meses un avance inesperado debido al COVID 19.

En el marco de esta evolución, los planificadores, arquitectos y pensadores urbanos, tienen la responsabilidad de comenzar a pensar en lo nocturno y diseñar espacios e intervenciones que faciliten las interacciones humanas a lo largo de todo el día. Las ciudades son organismos vivos las 24 horas del día y requieren de espacios gratuitos, abiertos y accesibles para vivir, trabajar y disfrutar. La noche es un espacio con infinitas posibilidades que deben ser explotadas y aprovechadas para el bien de todos. Aunque en ocasiones los planificadores y otros tomadores de decisión carecen de sensibilidad para comprender la escala humana a la que deben estar hechas las ciudades, la información y las distintas herramientas tecnológicas con las que cuentan pueden ayudar a enfrentarse mejor a los retos que tienen las ciudades (Mehmet, 2020). Las estrategias de ciudades nocturnas pueden aportar al cumplimiento del ODS 11 al promover ciudades sostenibles e incluyentes, con una oferta variada de bienes y servicios que aporte considerablemente al PIB de cada ciudad. 
El campo de la planificación urbana se debe robustecer, pues cada día adquiere mayor importancia en los territorios, no en vano ha quedado claro el rol que tienen respecto a la organización de las ciudades para ser más eficientes y garantizar, por lo menos a mediano plazo, una buena calidad de vida a sus ciudadanos. Lo importante dentro de esta planificación territorial es, sin duda, tener en cuenta que cada ciudad es distinta y cuenta con capacidades y habilidades únicas que deben valorarse a la hora de poner en marcha un proyecto. No hay fórmula mágica para todos los territorios y es ahí también donde el ciudadano inteligente juega un gran papel. Cada ciudad, junto con sus gobernantes y ciudadanos, debe definir las prioridades de acuerdo con sus fortalezas y visión de ciudad hacia el futuro. Lo anterior "se logra con estrategias prioritarias cuyo fin sea la locación eficiente de recursos públicos, para así promover la conformación de ciudades inteligentes en contextos geográficos heterogéneos" (Universidad del Rosario, 2020: 4).

No obstante, ante el avance e incertidumbre de la aplicación de las nuevas tecnologías de la comunicación y de la información, "se presentan nuevos y graves problemas como son: el aumento de la pobreza, y la exclusión social; el distanciamiento progresivo de los países ricos y pobres; la inseguridad y el terrorismo, unido a la creación de un sentimiento de desprotección y terror; los problemas de la degradación ambiental; la llamada brecha digital entre los poseedores de conocimientos, y los que no, a través de las nuevas tecnologías; la desafección y apatía políticas; el debilitamiento de la democracia; la precariedad laboral; el choque de civilizaciones y el multiculturalismo" (Romero, 2018: 33). Las ciudades tienen a la mano herramientas poderosas que deben saber aprovechar para mitigar estos obstáculos sociales, ambientales y económicos que perjudican a todos. La ciudad inteligente debe garantizar el bienestar para una gran mayoría de habitantes, si no es para todos.

Si bien el concepto de ciudad inteligente es bastante amplio, no hay que olvidar que hay muchas otras ideas que pueden complementar dicho significado. La biodiversidad, el espacio urbano-rural y el uso consciente de los recursos naturales, por ejemplo, son temas que han escapado a esta investigación, pero que, sin duda, pueden explorarse en mayor medida para robustecer lo que debe ser una ciudad inteligente. Lo que sí no puede olvidarse es que la ciudad inteligente tiene al ciudadano como centro, de ahí que su bienestar sea la métrica del éxito de una ciudad ideal (Rodríguez, 2019: 4). Los conceptos han ido evolucionando y se refuerzan 
constantemente para construir sobre lo construido y aprender de los errores conforme el mundo evoluciona y nuevas necesidades aparecen en el camino.

Las ciudades inteligentes del futuro serán las que guíen y lideren el crecimiento económico sostenible con la participación y el involucramiento de todos los actores que intervienen en el territorio. Sus líderes tendrán todas las herramientas disponibles para analizar los datos y tomar mejores decisiones, anticipándose a los problemas y resolviéndolos de forma coordinada con los recursos existentes (Cervantes, 2018). En síntesis, "las ciudades inteligentes promueven un ciclo virtuoso que produce no solo bienestar económico y social, sino también el uso sostenible de sus recursos con miras a elevar la calidad de vida a largo plazo" (Bouskela et al, 2016: 14).

\section{Referencias Bibliográficas}

Benítez, J. A. (2019). Alcaldías Nocturnas. Colombia, El Tiempo. http://blogs.eltiempo.com/ambienteurbano/2019/03/29/alcaldias-nocturnas/

Bouskela, M., Casseb, M., Bassi, S., De Luca, C. \& Facchina, M. (2016). La Ruta hacia las Smart Cities: Migrando de una gestión tradicional a la ciudad inteligente. Washington D. C.: Banco Interamericano de Desarrollo.

Bouskela, M. \& Elnir, H. (2018). Construyendo ciudades inteligentes en América Latina y el Caribe. Banco Interamericano de Desarrollo (BID). https://blogs.iadb.org/ciudadessostenibles/es/ciudadesinteligentes-smartcities-americalatinacaribe/

Cervantes, J. (2018). Smarter Cities by IBM.

Dirks, S. \& Keeling, M. (2009). A vision of smarter cities. How cities can lead the way into a prosperous and sustainable future. IBM Institute for Business Value. Executive Report. https://www.ibm.com/downloads/cas/2JYLM4ZA

eSMARTCITY. (2020). Woven City, la ciudad del futuro conectada y sostenible de Japón concebida como un laboratorio para desarrollar nuevas tecnologías. eSMARTCITY. https://www-esmartcityes.cdn.ampproject.org/c/s/www.esmartcity.es/2020/o1/10/wovencity-ciudad-futuro-conectada-sostenible-japon-concebida-

laboratorio-desarrollar-nuevas-tecnologias/amp

Garcia, J. (2018). "Planificando la smart city: participación ciudadana y financiación".

Harnish, C. (2016). Smarter Cities by IBM: The Road to Sustainable Societies. Better World International. 
Janka, L. (2016). Tiempo de observar a Jane Jacobs y a nuestras ciudades. Arquine. https://www.arquine.com/tiempo-de-observar-a-janejacobs-y-a-nuestras-ciudades/

Masud, F. (2019). The growing importance of the night-time economy. BBC. https://www.bbc.com/news/business-49348792

Mateu, C. (2018). ¿Qué son las ciudades inteligentes? Suelo Solar. https://suelosolar.com/newsolares/newsol.asp?id=12058

Mehmet, S. (2020). Project uses 'digital twins' to design more sustainable cities.

Intelligent

Transport.

https://www.intelligenttransport.com/transport-

news/98791/project-uses-digital-twins-to-design-more-sustainablecities/

Metro Cuadrado (2020). Las ciudades más inteligentes del mundo. Metro Cuadrado.

https://www.metrocuadrado.com/noticias/actualidad/lasciudades-mas-inteligentes-del-mundo-

3923?utm_source=eltiempo\&utm_medium=Enlace\&utm_campaig $\mathrm{n}=$ HomeCiudadesInteligentes

Naím, M. (2020). ¿Cómo serán las ciudades del futuro? El Tiempo. https://www.eltiempo.com/vida/ciencia/sokwoo-rhee-habla-sobrela-ciudades-del-futuro-con-moises-naim-505634

ONU Habitat. (2017). Los usos mixtos del suelo y sus beneficios. ONU Habitat. https://onuhabitat.org.mx/index.php/los-usos-mixtos-delsuelo-y-sus-beneficios

Organización de Naciones Unidas (2015). Objetivos de Desarrollo Sostenible. Organización de Naciones Unidas. https://www.un.org/sustainabledevelopment/es/cities/

Ospina, J. P. (2018). Ciudades Inteligentes en América Latina. Banco Interamericano de Desarrollo (BID). http://conexionintal.iadb.org/2018/11/27/267_e_ideas6/

Rodríguez, M. (2019). Nuestro planeta, nuestro futuro. Bogotá: Debate.

Romero, A. (2018). "El paradigma de las smart cities en el marco de la gobernanza urbana”. Gestión y Análisis de Políticas Públicas, 20, 29-35.

Sajhau, P. (2017). "IBM - Building sustainable cities through partnerships and integrated approaches". Field Actions Science Reports, 16, 5257.

Sánchez, C. (2019). Con oportunidades de negocios se potenciará la economía circular. Universidad Nacional de Colombia. http://ieu.unal.edu.co/medios/noticias-del-ieu/item/conoportunidades-de-negocio-se-potenciara-la-economia-circular

Secure Week (2017). ¿Cómo se están construyendo las ciudades más inteligentes del mundo? Secure Week.

Seijas, A. (2015a). El despertar de las ciudades nocturnas latinoamericanas. IQ Latino. https://iqlatino.org/2015/ciudades-nocturnas/ 
Seijas, A. (2015b). 5 beneficios de las ciudades 24 horas. Banco Interamericano de Desarrollo (BID). https://blogs.iadb.org/ciudades-sostenibles/es/ciudades-24-horas/

Seijas, A. (2018). Ciudades Nocturnas América Latina. https://www.academia.edu/40007093/Manifiesto_Latinoamerican o_de_Ciudades_Nocturnas

Seijas, A. \& Sound Diplomacy (2018). Una guía para gestionar su economía nocturna. London, Sound Diplomacy. https://static1.squarespace.com/static/593eb2a837c58172ed556cbb /t/5bf5dado70a6ad4582ec69e9/1542839152447/SoundDiplomacyNightTimeGuide-Spanish.pdf

Sostenibilidad (s.f.). ¿Qué es una Smart City? Top 5 ciudades inteligentes. Sostenibilidad https://www.sostenibilidad.com/construccion-yurbanismo/que-es-una-smart-city-top-5-ciudades-inteligentes/

Stagno, D. (2020). Economía circular, ciudades circulares: una alternativa sostenible para América Latina y el Caribe. Banco Interamericano de Desarrollo (BID). https://blogs.iadb.org/ciudadessostenibles/es/ciudades-circulares-economia-circularsostenibilidad-urbelac-europa-america-latina-caribe/

UN-Habitat. (2020). Future cities, new economy, and shared city prosperity driven by technological innovations. Nairobi: United Nations Human Settlements Programme (UN-Habitat).

Universidad del Rosario. (2020). Nota Tic Tank No. 2 Smart Cities: Tecnología para el desarrollo. Bogotá: Universidad del Rosario.

World Economic Forum \& Accenture Strategy. (2019). Harnessing the Fourth Industrial Revolution for the Circular Economy Consumer Electronics and Plastics Packaging. World Economic Forum. http://www3.weforum.org/docs/WEF_Harnessing_4IR_Circular_ Economy_report_2018.pdf

\footnotetext{
${ }^{1}$ Una de las grandes lecciones que ha dejado la pandemia generada por el COVID-19 ha sido precisamente la importancia que tienen los alcaldes en la toma de decisiones a la hora de enfrentar los problemas locales.
} 